\title{
Pelle and Tube contribute to the Toll pathway-dependent antimicrobial peptide production in the red flour beetle, Tribolium castaneum (Coleoptera: Tenebrionidae)
}

\author{
KAKERU YOKOI $^{1,2}$ (D), DAIKI KATO ${ }^{1}$ and KEN MIURA ${ }^{1}$ \\ ${ }^{1}$ Applied Entomology Laboratory, Graduate School of Bioagricultural Sciences, Nagoya University, Furo-cho, Chikusa, Nagoya \\ 464-8601, Japan; e-mails: daiki@agr.nagoya-u.ac.jp, k-miura@agr.nagoya-u.ac.jp \\ ${ }^{2}$ Insect Design Technology Group, Division of Insect Advanced Technology, Institute of Agrobiological Sciences, NARO, Owashi, \\ Tsukuba 305-8643, Japan; e-mail: yokoi123@affrc.go.jp
}

Key words. Innate immunity, RNA interference

\begin{abstract}
Insects are solely dependent on an innate immune system. Antimicrobial peptide production is the main immune response of insects. The molecular mechanisms underlying this reaction in Drosophila melanogaster involves the induction of antimicrobial peptide genes, which is regulated by the Toll and IMD pathways. The Toll pathway is mainly activated by fungi or Gram-positive bacteria and the IMD pathway by Gram-negative bacteria. In terms of comparative immunology, we investigated the antimicrobial peptide production system in the beetle, Tribolium castaneum, which differs from that in $D$. melanogaster. To obtain a more detailed understanding, we examined whether Pelle and Tube, orthologues of which in D. melanogaster are the Toll pathway components, contributed to antimicrobial peptide production and immune reactions. These two genes were not induced by challenges from any type of microbe, which in this case were Gram-positive bacteria, -negative bacteria and an eukaryote. Using Pelle and Tube knockdown pupae, it was demonstrated that Pelle and Tube are involved in the induction of Cec2 as a representative Toll pathway-dependent gene in $T$. castaneum by Gram-positive and -negative bacteria and eukaryote challenges. Furthermore, neither Pelle nor Tube contributed to immune defences against two entomopathogenic bacteria. These results, taken together with our previous findings, led to the conclusion that the Toll pathway immune signaling reported in $D$. melanogaster indeed occurs in $T$. castaneum, and the gene sets involved in Toll signal transduction in T. castaneum did not differ significantly from those in $D$. melanogaster, but transduced immune signals to challenges from Gram-positive bacteria, -negative bacteria and an eukaryote, which differed from those in $D$. melanogaster.
\end{abstract}

\section{INTRODUCTION}

Insects do not possess an adaptive immune defense system; they rely solely on an innate immune system. Insect immune reactions consist of both cellular and humoral immune reactions. While the cellular reaction is mediated by haemocytes, the humoral reaction produces effector molecules, such as antimicrobial peptides (AMPs) and melanin, the functions of which are to protect the host from microbial attacks (Hultmark, 2003; Hillyer, 2016). The regulatory system of AMP production has been extensively examined in Drosophila melanogaster (Meigen) and mainly involves two signaling pathways, the IMD and Toll pathways (Ferrandon et al., 2007; Lemaitre \& Hoffmann, 2007).

In D. melanogaster, the Toll pathway is activated by lysine-type peptidoglycans derived from Gram-positive bacteria or $\beta-1,3$ glucan from fungi or yeast, which are recognized by the Gram-negative binding protein 1 (GNBP1) and peptidoglycan recognition protein SA (PGRP-SA) complex and GNBP3, respectively (Gottar et al., 2006;
Wang et al., 2006). Signals from sensor proteins are then transduced through a serine protease cascade, leading to the proteolytic activation of the Toll ligand, Spätzle (Spz) (Weber et al., 2003). Two Tolls with activated Spz in turn activate the intracellular signaling pathway, the Toll pathway. After signal transduction, the protein MyD88 is recruited through the TID domain, which then recruits another adapter protein, Tube and the kinase Pelle (Medzhitov et al., 1998; Horng \& Medzhitov, 2001). These three proteins ultimately form heterotrimer complexes, which phosphorylate and degrade the inhibitor of $\mathrm{NF}-\kappa \mathrm{B}$, cactus by an unknown mechanism (Horng \& Medzhitov, 2001; Valanne et al., 2011). Consequently, the NF- $\kappa \mathrm{B}$ transcription factor Dif or Dorsal translocate into the nucleus and induces a number of immune effector genes, including several AMPs (e.g., Drosomycin) (Ip et al., 1993; Lemaitre et al., 1996). The IMD pathway is mainly activated by Meso-diaminopimelic acid-type peptidoglycans derived from Gramnegative bacteria through PGRP-LC, and an immune 
signal is transmitted (Choe et al., 2002, 2005; Kaneko et al., 2006). The signal transduces through IMD protein and intracellular signaling proteins consisting of the IMD pathway (Hu \& Yang, 2000; Guntermann \& Foley, 2011). After signal transduction to the end of the IMD pathway, another $\mathrm{NF}-\kappa \mathrm{B}$ class transcription factor, Relish, is processed and translocated into the nucleus, and Relish induces several effector genes, including some AMPs (e.g., Diptericin) (Stoven et al., 2003; Ferrandon et al., 2007; Lemaitre \& Hoffmann, 2007; Kleino \& Silverman, 2014).

The red flour beetle, Tribolium castaneum (Herbst), is a model coleopteran insect for which the whole genome sequence and gene set data are available (Zou et al., 2007; Tribolium Genome Sequencing Consortium et al., 2008). Furthermore, systemic RNA interference (RNAi) is induced in beetles by an injection of double-stranded RNA (dsRNA) (Tomoyasu et al., 2008). Therefore, this beetle is very useful for genomic and molecular biological research.

In terms of comparative immunology and entomology, a more detailed understanding of the immune systems of non-Drosophila species is required. Due to the importance and features of $T$. castaneum described above, we investigated its immune system, particularly the regulation of AMP production. Our findings revealed that AMP genes in $T$. castaneum are induced by several types of microbe, namely, Gram-positive and -negative bacteria as well as yeast (Yokoi et al., 2012b). By using knockdown pupae of $I M D$ and $M y D 88$, both of which are adapter genes in the IMD and Toll pathways, respectively, nine T. castaneum AMP genes were divided into four groups: group I (IMD pathway-dependent and high level induction $6 \mathrm{~h}$ after a challenge), group II (Toll and IMD pathway-dependent and high level induction $6 \mathrm{~h}$ after a challenge), group III (Toll pathway-dependent and sustained induction 6 to 24 $\mathrm{h}$ after a challenge), and group IV (neither Toll nor IMD pathway-dependent). We further investigated AMP induction systems using AMP genes as a read-out for the two signaling pathways. Our findings revealed that $M y D 88$ Dif (Dorsal) and IMD-Relish transduced the same signals, while PGRP-LA and other PGRPs contributed to the recognition of both Gram-positive and -negative bacteria, which activated the IMD pathway (Yokoi et al., 2012a). We also demonstrated, using knockdown assessments of $M y D 88, I M D$ and the other IMD intracellular-signaling components, DREDD and FADD, that crosstalk in immune signals between the Toll and IMD pathways ["crosstalk" means that signals transducing a signaling pathway transduce or affect other signaling pathways. In this study, "crosstalk" means immune signals aroused from upstream of Toll or IMD pathway may affect or transduce another pathway, which is a feature of the IMD and Toll pathways in T. castaneum (Yokoi et al., 2022)]. In the present study, in order to obtain insights into the IMD and Toll pathways in T. castaneum, we investigated whether Tube and Pelle, which are Toll pathway components, are involved in the immune reaction mediated by the Toll or IMD pathway using RNAi. The results indicate that Pelle and Tube function as Toll pathway signal transducers and do not con- tribute to signal crosstalk, which is reported occurring in $F A D D, D R E D D$ and IMD knockdown pupae (Yokoi et al., 2012b, 2022). Furthermore, neither Pelle nor Tube are involved in immune defences against two entomopathogenic bacteria, which is consistent with $M y D 88$.

\section{MATERIALS AND METHODS}

\section{Insect rearing}

T. castaneum was reared on whole wheat flour at $30^{\circ} \mathrm{C}$ in the dark. Pupae for experiments were staged as described in our previous study (Yokoi et al., 2012b).

\section{Microbes and injections}

Heat-killed Escherichia coli DH5a, Micrococcus luteus ATCC4698 and Saccharomyces cerevisiae S288C were prepared as elicitors of immune reactions, which are representative microbes of Gram-negative bacteria, Gram-positive bacteria and eukaryotes, respectively. The preparation of these microbes is previously described (Yokoi et al., 2012b). Fifty nanolitres of suspensions containing E. coli, M. luteus or S. cerevisiae, which are equivalent to $2.9 \times 10^{8}, 2.9 \times 10^{7}$, and $6.3 \times 10^{6} \mathrm{cells} / \mathrm{ml}$, respectively, were injected into untreated or previously treated with dsRNA 3 day-old pupae using Nanoject II (Drummond Scientific company, Broomall, PA, USA). In addition, living Enterobacter cloacae and Bacillus subtilis were used in the survival assay as described in our previous study (Yokoi et al., 2012a).

\section{Gene sequences}

T. castaneum gene sequences used in the present study from reference genome version Tcas 2.0 were retrieved from Tcas2.0/annotation/TCGleanPrediction data (URL: ftp://ftp. hgsc.bcm.edu/Tcastaneum/) available at "BCM-HGSC data" in the "Red Flour Beetle Genome Project" site (URL: https:// www.hgsc.bcm.edu/arthropods/red-flour-beetle-genome-project) (Zou et al., 2007; Tribolium Genome Sequencing Consortium, 2008): Pelle (GLEAN_15365); Tube (Gene ID in gene set data: GLEAN_11895); Attacin 1 (Att1) (GLEAN_07737); (GLEAN_07739); Cecropin 2 (Cec2) (GLEAN_00499); Coleoptericin 1 (Coll) (GLEAN_05093 and GLEAN_10517: Col1 was split into two genes in gene set data); Defensin 3 (Def3) (GLEAN_12469); IMD (GLEAN_10851); and the normalizer gene for qRT-PCR analyses, ribosomal protein L32 (RPL32) (GLEAN_06106).

\section{RNA extraction and quantitative RT-PCR (qRT-PCR)}

Total RNA was extracted from whole $T$. castaneum pupae with TRIZOL reagent (Invitrogen, Waltham, MA, USA) and spectrophotometric scanning was used to assess the quality of the extracted RNA. According to manufacturer's instructions, $0.5 \mu \mathrm{g}$ of total RNA was converted into first-strand cDNA using PrimeScript RT Reagent Kit with gDNA Eraser (TAKARA. Kusatsu, Shiga, Japan), and qRT-PCR was performed using first-strand cDNA and a primer pair designed for each target gene with the SYBR Premix Ex Taq Perfect Real Time Kit Tli RNAaseH Plus (TAKARA) and Thermal Cycler Dice Real Time System (Model TP800, TAKARA). The sequences of primer pairs used for qRTPCR are shown in Supplementary Table 1. qRT-PCR conditions and methods for calculating levels of mRNA relative to RPL32 are described in our previous study (Yokoi et al., 2012b).

\section{RNAi}

RNAi was performed as described in our previous study (Yokoi et al., 2012b). Briefly, the dsRNA of each gene was synthesized using the cDNA template with T7 RNA polymerase promoter sequences on both sides by the MEGAscript RNAi Kit (Ambion, 
A

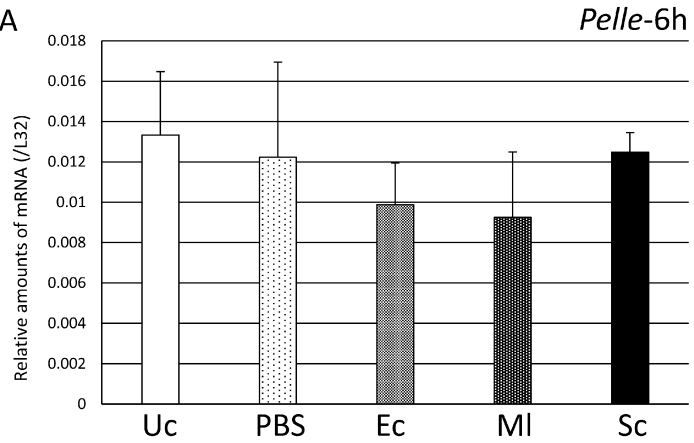

B

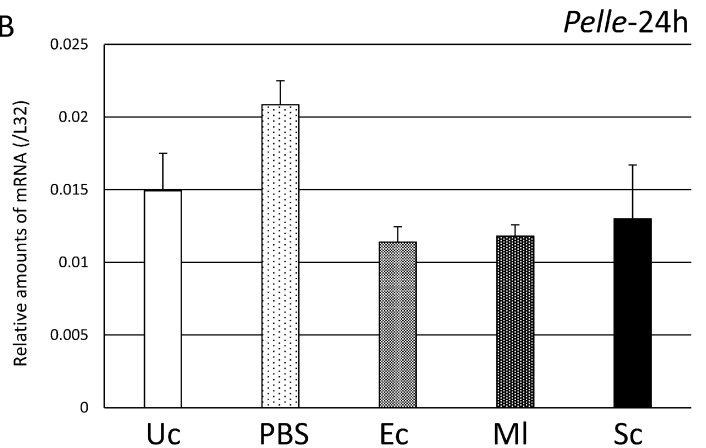

C

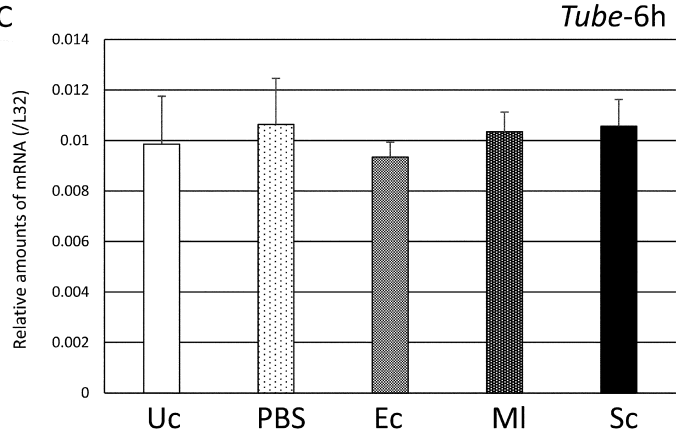

D

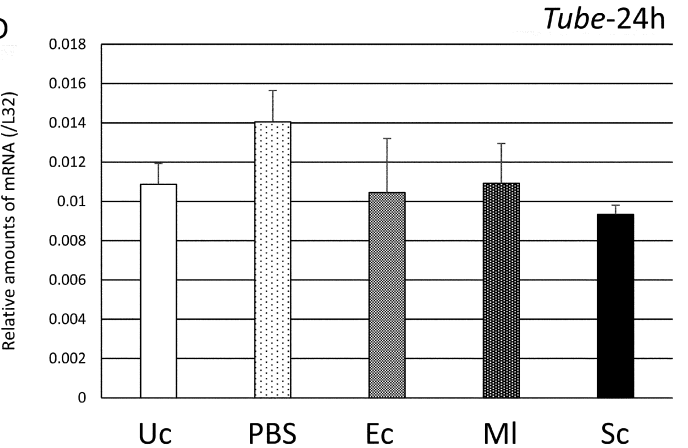

Fig. 1. Effects of microbial challenges on the relative amounts of mRNA of Pelle and Tube. Heat-killed cell suspensions of $E$. coli (Ec), $M$. luteus (MI), or S. cerevisiae (Sc) were injected into day 3 pupae, and the amounts of mRNA of Pelle post challenge $6 \mathrm{~h}(\mathrm{~A}), 24 \mathrm{~h}(\mathrm{~B})$, Tube post challenge $6 \mathrm{~h}(\mathrm{C})$ and $24 \mathrm{~h}(\mathrm{D})$ were measured using qRT-PCR 6 and $24 \mathrm{~h}$ after the challenges. Unchallenged (Uc) and PBS-injected day 3 pupae (PBS) were the negative controls. The amounts of mRNA of the two genes are shown relative to those of $R p L 32$ levels in the same samples. Experiments with 3 animals were independently repeated at least three times, and each column is the mean \pm S.D. Student's $t$-tests of the Pelle and Tube mRNA values in Uc pupae versus PBS-, Ec-, MI- Sc- injected pupae were done and none of the combinations were significant $(P>0.05)$.

Waltham, MA, USA). Sequences of primer pairs for the cDNA template are shown in Supplementary Table 2. One hundred nanograms of each dsRNA was injected into day 0 pupae, which were then kept in the dark at $30^{\circ} \mathrm{C}$ for $72 \mathrm{~h}$. For preparation of negative controls, dsRNA of maltose binding protein $E$ (malE) was injected into day 0 pupae. These pupae were used in microbe challenges or for qRT-PCR.

\section{Survival assay}

Fifty nanolitres of live E. cloacae and B. subtilis suspensions, the $\mathrm{A}_{600}$ values of which were 0.1 and 4.0, respectively, were injected into dsRNA-treated day 3 pupae using Nanoject II. Surviving pupae were counted every $24 \mathrm{~h}$. Data are shown in Kaplan-Meier plots and P-values were calculated using the Gehan-Breslow-Wilcoxon test in R ("survival" and "MASS" library with RStudio Version 1.3.1073).

\section{RESULTS}

\section{Pelle and Tube were not induced by microbial challenges}

Some of the genes involved in immune reactions are generally induced by microbial challenges (De Gregorio et al., 2001, 2002) and several immune-related genes in T. castaneum are induced by microbial challenges (Yokoi et al., 2012a, b; Koyama et al., 2015). Therefore, we investigated whether Pelle and Tube in T. castaneum were induced 6 or $24 \mathrm{~h}$ after microbial challenges by $E$. coli, $M$. luteus or $S$. cerevisiae. As shown in Fig. 1, the amounts of Pelle and Tube mRNA in unchallenged pupae did not differ significantly from those in PBS- or any microbe-injected pupae, suggesting that neither Pelle nor Tube were induced by any microbial challenge.

\section{Knockdown effects of Pelle and Tube by RNAi}

To confirm the knockdown effects against Pelle and Tube by RNAi, the amounts of mRNA of Pelle and Tube in dsRNA-injected pupae were assessed using qRT-PCR (pupae treated with malE dsRNA were used as controls). The amounts of mRNA of Pelle after $72 \mathrm{~h}$ were markedly lower in pupae treated with Pelle dsRNA than in control pupae (Fig. 2A). Furthermore, the amounts of mRNA of Tube after $72 \mathrm{~h}$ were markedly lower in pupae treated with Tube dsRNA than in control pupae (Fig. 2B). These results indicate that dsRNA-treated pupae are suitable for use in assessments of Pelle and Tube functions.

\section{Neither Pelle nor Tube were involved in Group I AMP gene induction (IMD pathway-dependent)}

To assess whether Pelle and Tube contribute to IMD or Toll pathway-dependent immune signaling, the amounts of mRNA of several AMP genes as read-outs of the Toll or IMD pathway in Pelle or Tube knockdown pupae challenged by several microbes were measured. The amounts of mRNA of Attl and Coll, as representative IMD pathway-dependent AMP genes (group I AMP genes) (Yokoi et al., 2012b), were measured in Pelle or Tube knockdown plus control pupae (pre-treated with malE dsRNA) 6 and 

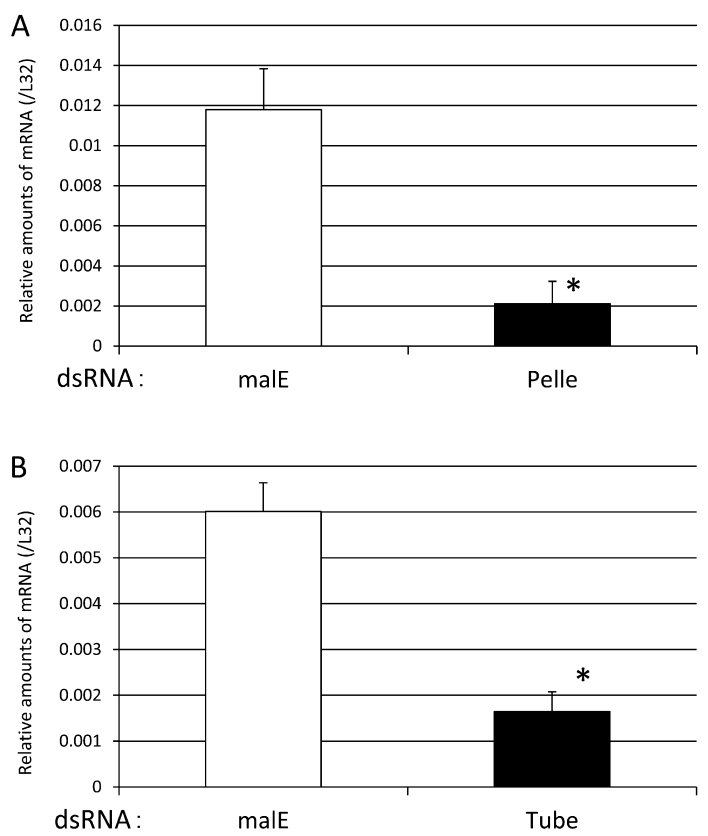

Fig. 2. Efficiency of RNAi-mediated gene knockdown on Pelle and Tube. Day 0 pupae were injected with $100 \mathrm{ng}$ of Pelle, Tube or control malE dsRNA and the levels of mRNA of Pelle (A) and Tube (B) relative to those of $R p L 32$ were calculated after $72 \mathrm{~h}$ using qRT-PCR. Experiments were independently repeated at least three times with 3 animals, and the mean \pm S.D. is shown. Asterisks indicate $P<0.05$ versus controls based on Student's $t$-test.

$24 \mathrm{~h}$ after being challenged by either E. coli, M. luteus or $S$. cerevisiae (Figs 3 and 4). The knockdown of Pelle or Tube only slightly affected the induction of Att1 by E. coli after 6 h (Fig. 3A), while after $24 \mathrm{~h}$ it was not altered (Fig. 3B). Regarding the M. luteus challenge, the induction of Att1 after $6 \mathrm{~h}$ was very slightly affected by the Pelle or Tube knockdown (Fig. 3C), whereas that after $24 \mathrm{~h}$ was not (Fig. 3D), which was similar to the results obtained after the $E$. coli challenge. Neither the Pelle nor Tube knockdown affected the induction of Att 1 by the $S$. cerevisiae challenge after 6 and $24 \mathrm{~h}$ (Fig. 3E and 3F).

The amounts of mRNA of Coll $6 \mathrm{~h}$ after the challenge by E. coli were significantly lower in Pelle knockdown pupae than in control pupae, whereas those in Tube knockdown pupae were not (Fig. 4A). The amounts of mRNA of Coll in Pelle or Tube knockdown pupae $24 \mathrm{~h}$ after the $E$. coli challenge were not significantly different from those in control pupae (Fig. 4B). In the case of the M. luteus challenge, no significant differences were recorded in the amounts of mRNA of Coll between Pelle or Tube knockdown pupae and control pupae 6 and $24 \mathrm{~h}$ after the challenges (Fig. 4C and 4D). The amount of mRNA of Coll in Pelle or Tube knockdown pupae challenged with $S$. cerevisiae did not significantly differ from those in control pupae (Fig. 4E and 4F). These results indicate that neither Pelle nor Tube contributed to Group I induction by any of the microbes used.

\section{Neither Pelle nor Tube were involved in Group II AMP gene induction (IMD and Toll pathway- dependent)}

We assessed the effects of the knockdown of Pelle and Tube, as representative Toll and IMD pathway-dependent AMP gene (group II AMP gene), on the induction of Def3 by microbe challenges in $T$. castaneum pupae (Yokoi et al., 2012b). The amounts of mRNA of Def3 6 and $24 \mathrm{~h}$ after the challenge with $E$. coli were significantly lower in Pelle knockdown pupae than in control pupae, but not in Tube knockdown pupae (Fig. 5A and 5B). The amounts of mRNA of Def3 6 and $24 \mathrm{~h}$ after the challenge with $M$. luteus did not differ significantly between Pelle or Tube knockdown pupae and control pupae (Fig. 5C and 5D). Furthermore, the abundant amounts of mRNA of Def 3 in Pelle or Tube knockdown pupae 6 and $24 \mathrm{~h}$ after the challenge with $S$. cerevisiae did not differ significantly from those in control pupae (Fig. 5E and 5F). These results indicate that Pelle was involved in the induction of Def3, but only when challenged by $E$. coli, whereas Tube did not contribute to its induction by microbe infections.

\section{Pelle and Tube contributed to Group III AMP gene induction (Toll pathway-dependent)}

To establish whether Pelle and Tube are involved in Cec2 gene induction as representative Toll pathway-dependent AMP genes (group III AMP genes), which showed higher induction levels $24 \mathrm{~h}$ than $6 \mathrm{~h}$ after a challenge (Yokoi et al., 2012b), the amounts of mRNA of Cec2 in Pelle or Tube knockdown pupae and control pupae (pre-treated with malE dsRNA) 6 and $24 \mathrm{~h}$ after challenge by E. coli, M. luteus or $S$. cerevisiae were measured with qRT-PCR, and the amounts of $\mathrm{Cec} 2 \mathrm{mRNA}$ in these knockdown pupae were compared with those in control pupae (Fig. 6). The amounts of mRNA of Cec 2 in Pelle or Tube knockdown pupae $6 \mathrm{~h}$ after the challenge with $E$. coli were approximately $50 \%$ of that in the control pupae (Fig. 6A). Amounts of Cec2 mRNA in Pelle and Tube knockdown pupae $24 \mathrm{~h}$ after the challenge with E. coli were approximately 30 and 50\%, respectively, of that in the control pupae (Fig. 6B). Six hours after the M. luteus challenge, the amounts of $\mathrm{Cec} 2$ mRNA in Pelle knockdown pupae were lower, but not significantly so, than those in control pupae, whereas those of Tube knockdown pupae were significantly lower (Fig. 6C). Levels of Cec 2 mRNA in Pelle and Tube knockdown pupae $24 \mathrm{~h}$ after the $M$. luteus challenge were markedly lower than those in control pupae, and the extent of the reductions in Pelle and Tube knockdown pupae were approximately 30 and $50 \%$, respectively, which were similar to that recorded in pupae post $E$. coli challenge $24 \mathrm{~h}$ (Fig. 6D). Six hours after the challenge with $S$. cerevisiae, Pelle and Tube knockdown pupae both had smaller amounts of mRNA of Cec2 than the control pupae (Fig. 6E). Twentyfour hours after the $S$. cerevisiae challenge, the amounts of mRNA of Cec2 were significantly lower in Pelle and Tube knockdown pupae than in control pupae (Fig. 6F). These 
A

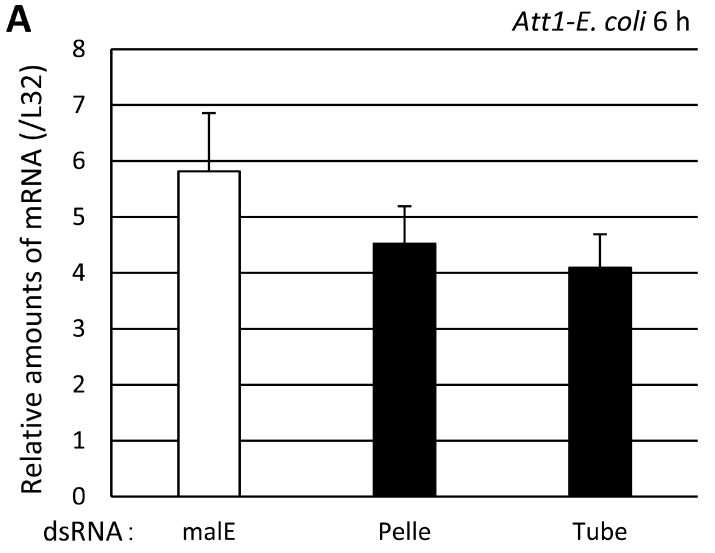

C

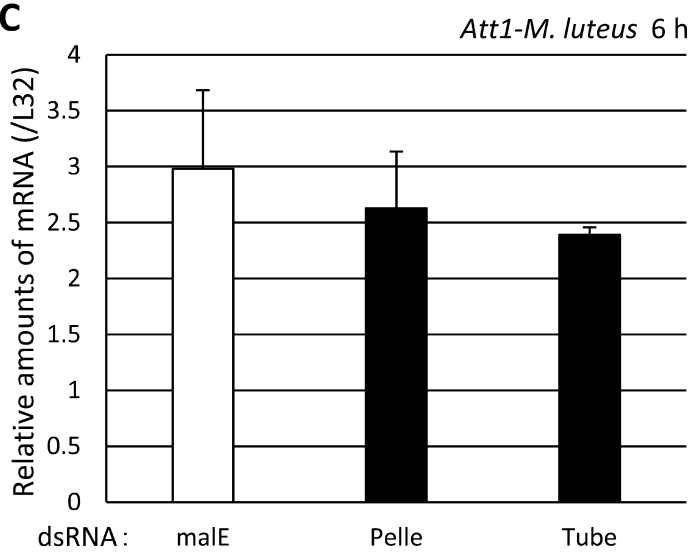

$\mathbf{E}$

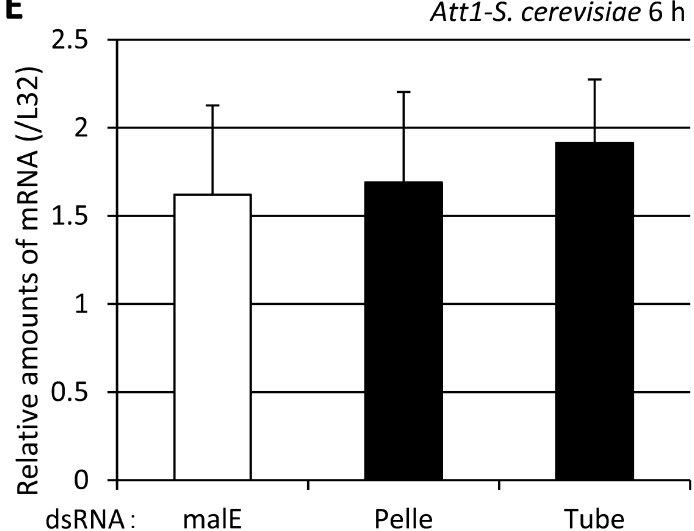

B

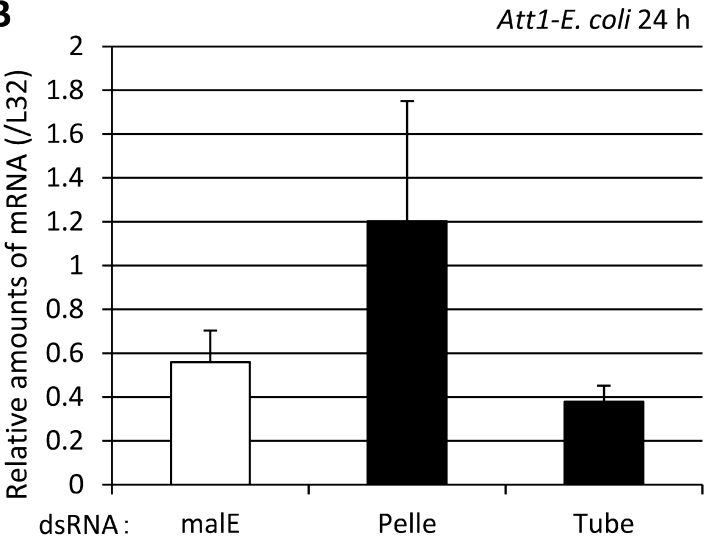

D

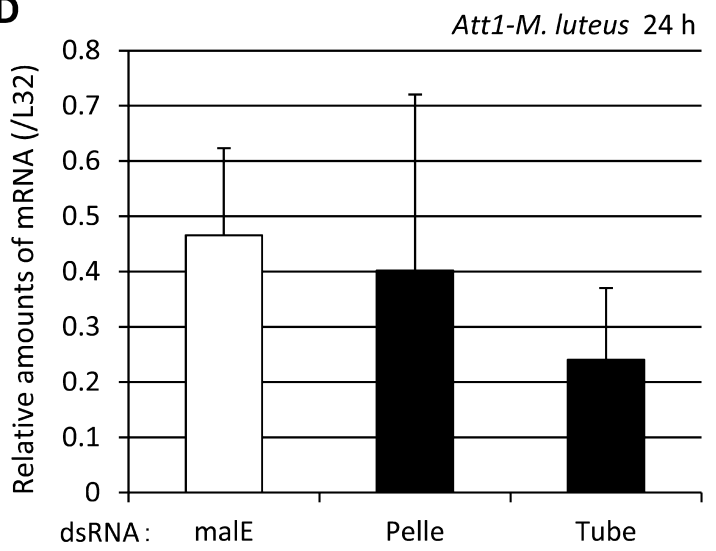

$\mathbf{F}$

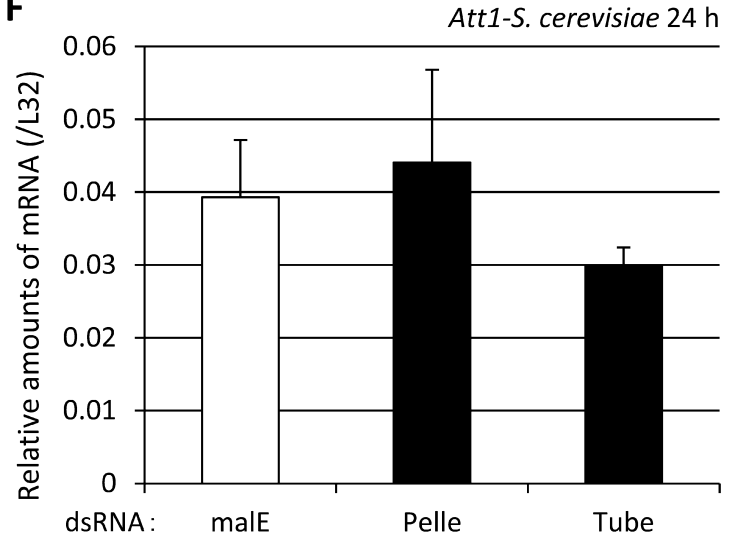

Fig. 3. Effects of the knockdown of Pelle and Tube on the microbial induction of the group I AMP gene, Att1. 100ng dsRNAs of Pelle, Tube and malE were injected into day 0 pupae. $72 \mathrm{~h}$ later, a heat-killed cell suspension of $E$. coli (A and $B), M$. luteus (C and D) or $S$. cerevisiae ( $\mathrm{E}$ and $\mathrm{F}$ ) was then injected into dsRNA-treated pupae. The relative amounts of mRNA of $A t t 1$ to RpL32 were assessed $6 \mathrm{~h}(\mathrm{~A}, \mathrm{C}$ and $\mathrm{E})$ and $24 \mathrm{~h}(\mathrm{~B}, \mathrm{D}$ and $\mathrm{F})$ after the microbial challenges. The results are the mean \pm S.D. Experiments were independently repeated at least three times with three animals each.

results indicate that Pelle and Tube were both involved in $\operatorname{Cec} 2$ (Toll pathway-dependent) gene induction by the three types of microbial challenges used.

\section{Neither Pelle nor Tube contributed to the immune defense against entomopathogenic bacteria}

To determine whether Pelle and Tube are involved in the immune defences of $T$. castaneum against invading microbes, we carried out survival assays using two model pathogenic bacterial species, E. cloacae and B. subtilis. Pupae pre-treated with Pelle, Tube, or malE dsRNA were injected with defined doses of live E. cloacae or B. subtilis and the proportions surviving were calculated every 24 h. When challenged with E. cloacae, the survival curves of Pelle and Tube knockdown pupae did not differ significantly from that of control pupae $(P>0.5)$ (Fig. 7A). When challenged with $B$. subtilis, more Pelle and Tube knockdown pupae survived than control pupae 24 to $72 \mathrm{~h}$ after the challenge (Fig. 7B). However, the survival curves of Pelle and Tube knockdown pupae did not differ significantly from that of control pupae. 
A

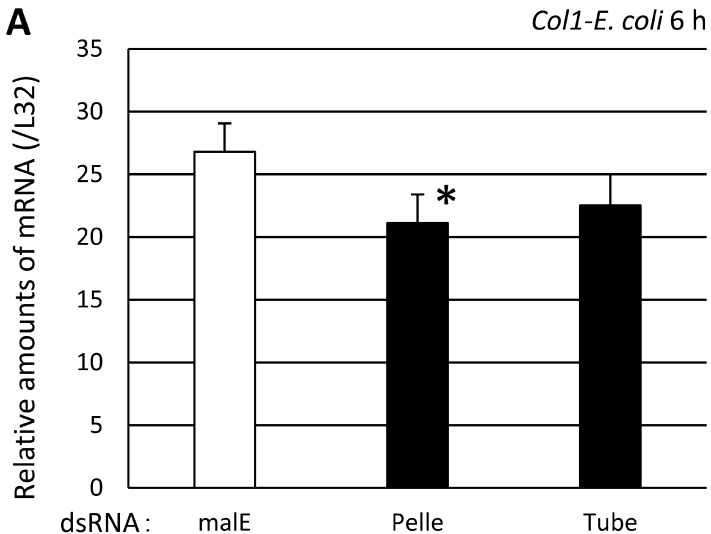

C

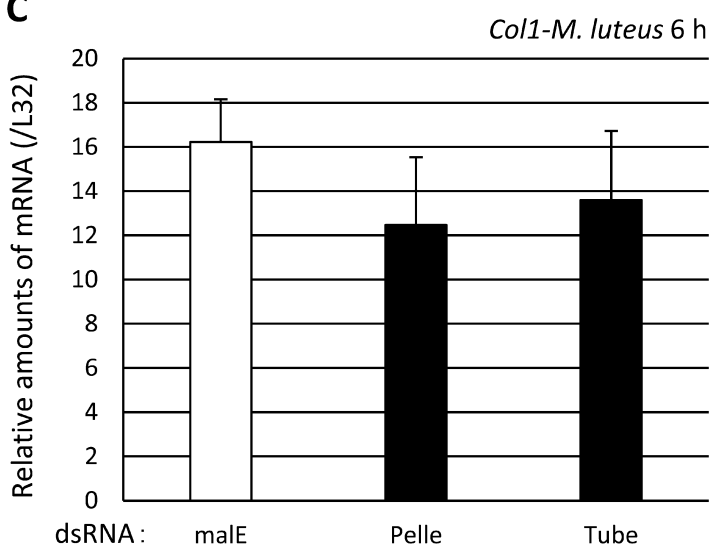

E

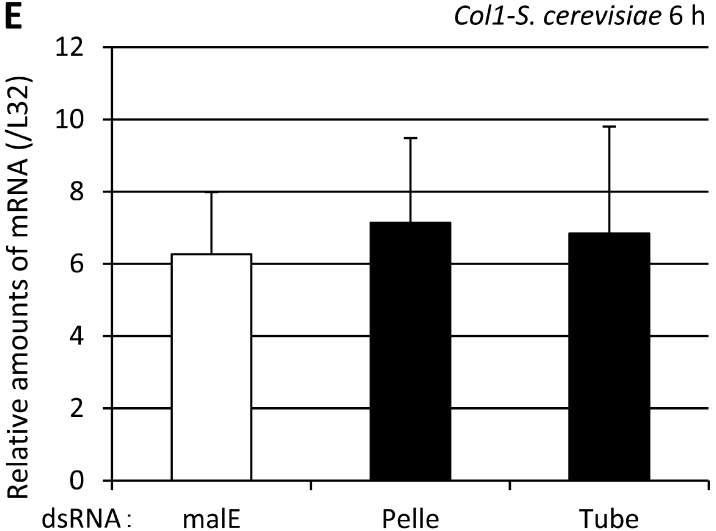

B

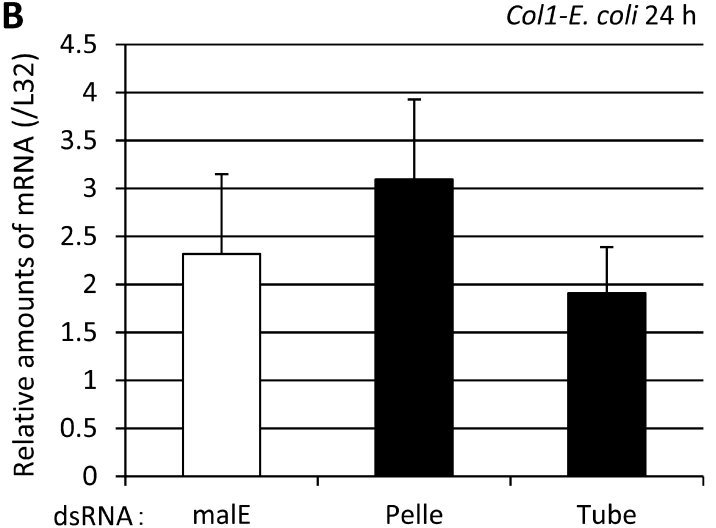

D

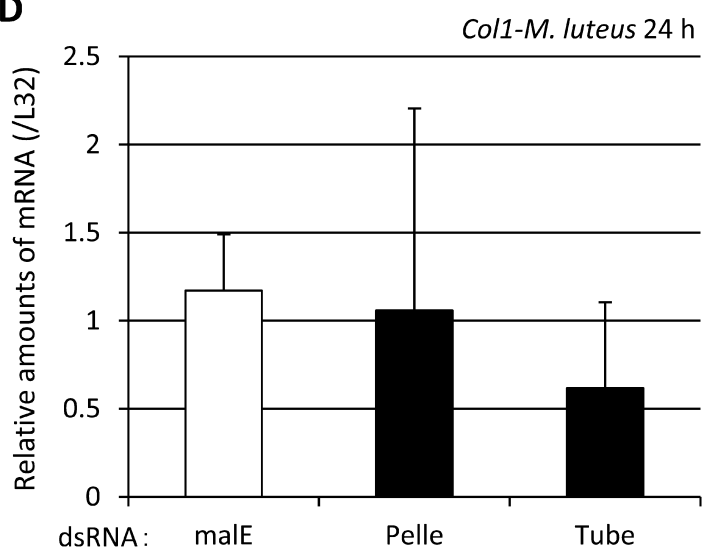

$\mathbf{F}$

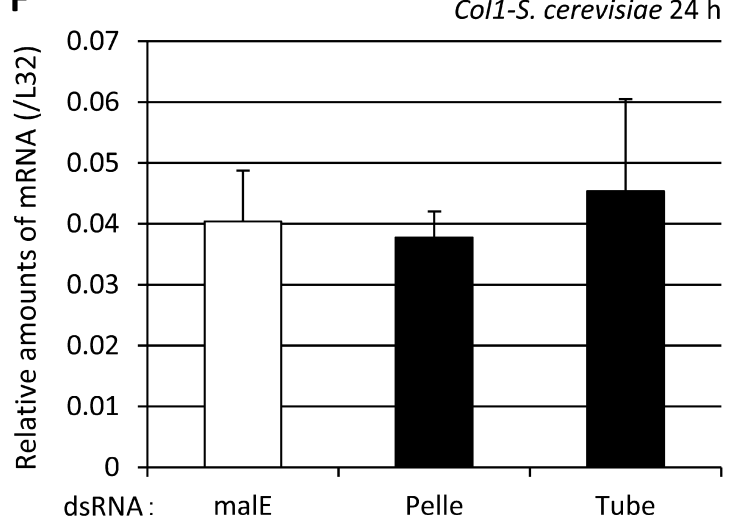

Fig. 4. Effects of the knockdown of Pelle and Tube on the microbial induction of the group I AMP gene, Col1. 100 ng dsRNAs of Pelle, Tube, and malE were injected into day 0 pupae. $72 \mathrm{~h}$ later, a heat-killed cell suspension of $E$. coli (A and B), M. luteus (C and D) or $S$. cerevisiae (E and F) was injected into dsRNA-treated pupae. The relative amounts of mRNA of Col1 to RpL32 were assessed $6 \mathrm{~h}$ (A and $\mathrm{E})$ and $24 \mathrm{~h}(\mathrm{~B}, \mathrm{D}$ and $\mathrm{F})$ after the microbial challenges. The results are the mean \pm S.D. Experiments were independently repeated at least three times with three animals each. Asterisks indicate $P<0.05$ versus malE control pupae based on Student's $t$-tests.

\section{DISCUSSION}

In the present study, we investigated whether Pelle or Tube in T. castaneum play a role in the production of AMP, one of the main immune reactions in insects, using RNAi. The results indicate that neither Pelle nor Tube was induced by challenges from three microbes, namely, heatkilled E. coli (Gram-negative bacteria) M. luteus (Grampositive bacteria) and $S$. cerevisiae (eukaryote), and Pelle and Tube mainly contributed to the induction of $\mathrm{Cec} 2$ by these microbe challenges, which was mainly regulated by the Toll pathway (Yokoi et al., 2012b). Furthermore, we showed that neither Pelle nor Tube were involved in host immune defenses against two entomopathogenic bacteria, E. cloacae and B. subtilis.

In previous studies on Drosophila, several immune-related genes are reported induced by microbial challenges and contribute to immune reactions (De Gregorio et al., 2001, 2002). In T. castaneum, Dif2 and Rel, which are NF- $\kappa \mathrm{B}$ transcription factors, are mainly involved in Toll pathway- and IMD pathway-dependent AMP gene inductions, which are induced by challenges by the microbes: $E$. 
A

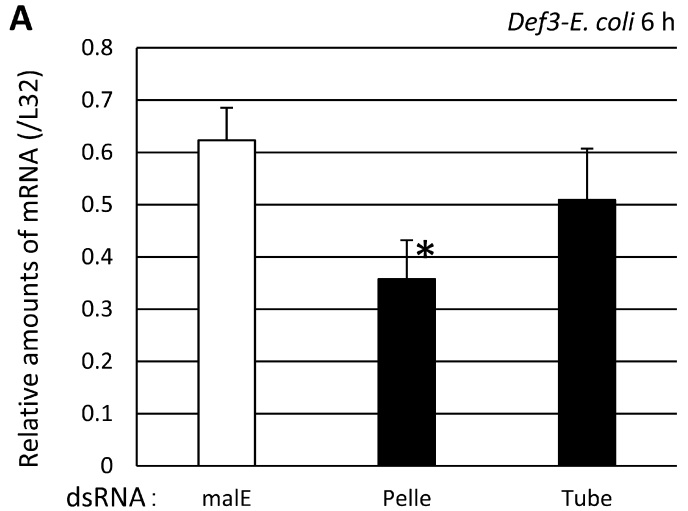

C

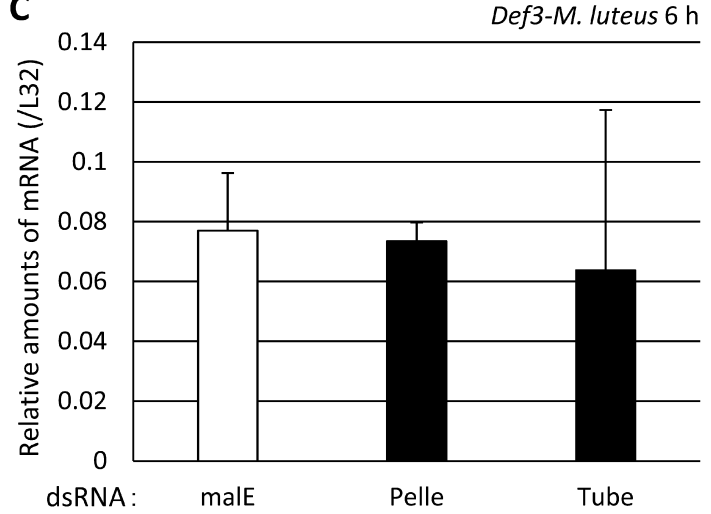

E

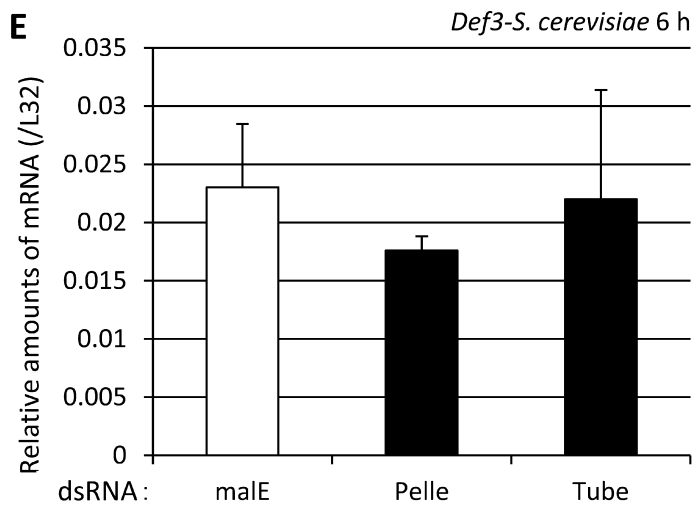

B

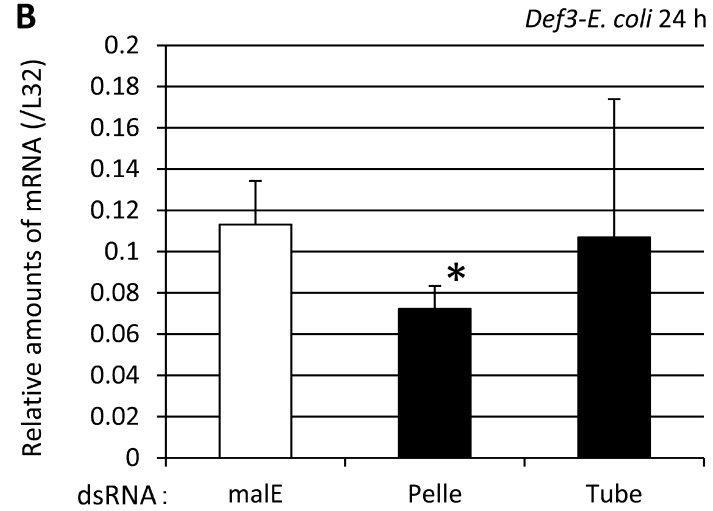

D

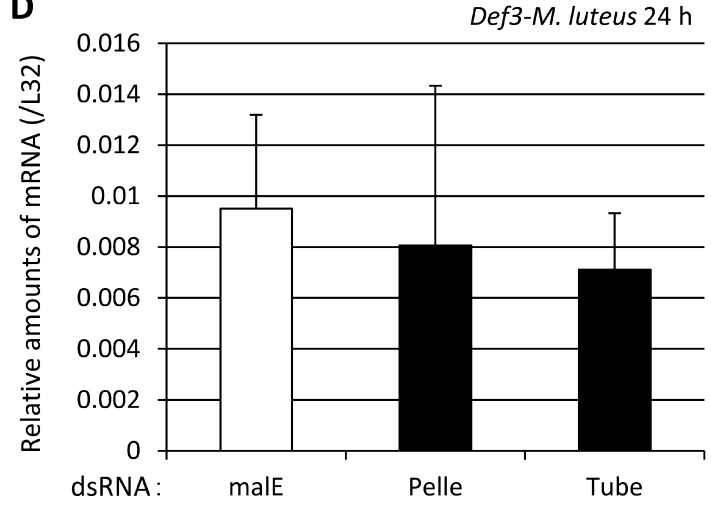

E

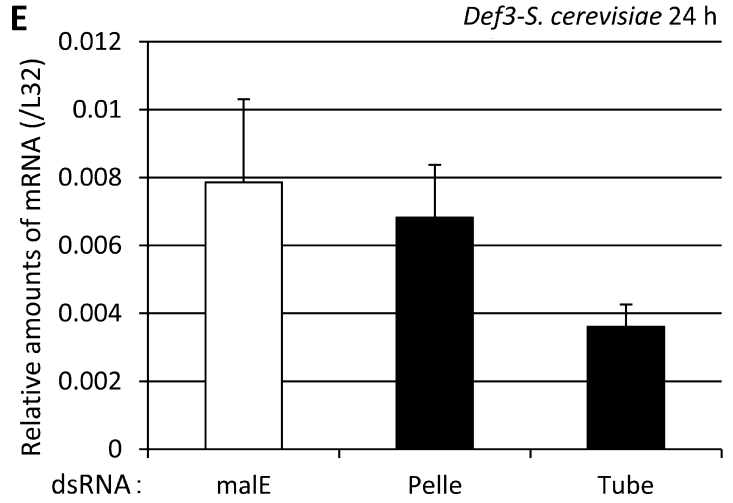

Fig. 5. Effects of the knockdown of Pelle and Tube on the microbial induction of the group II AMP gene, Def3. 100ng dsRNAs of Pelle, Tube and malE were injected into day 0 pupae. $72 \mathrm{~h}$ later, a heat-killed cell suspension of $E$. coli (A and $B)$, M. luteus (C and D) or $S$. cerevisiae ( $\mathrm{E}$ and $\mathrm{F}$ ) was injected into dsRNA-treated pupae. The relative amounts of mRNA of Col1 to RpL32 were assessed $6 \mathrm{~h}(\mathrm{~A}, \mathrm{C}$ and $\mathrm{E})$ and $24 \mathrm{~h}(\mathrm{~B}, \mathrm{D}$ and $\mathrm{F})$ after the microbial challenges. The results are the mean \pm S.D. Experiments were independently repeated at least three times with three animals each. Asterisks indicate $P<0.05$ versus malE control pupae based on Student's $t$-tests.

coli, M. luteus and S. cerevisiae challenges (Yokoi et al., 2012a, b). Among PGRP family of genes, PGRP-LA, $-L C$ and $-L E$, which function as sensors for the IMD pathway, are induced by $E$. coli and $M$. luteus challenges (Koyama et al., 2015). Although PGRP-SA and $-S B$ are induced, their functions in AMP gene induction remain unknown. $D R E D D$ and $F A D D$, which are components of intracellular-signaling part of the IMD pathway that are mainly involved in IMD pathway-dependent AMP gene induction, are not induced by any of the three microbes described above (Yokoi et al., 2022). In the present study, we showed that neither Pelle nor Tube was induced by any of the three microbes. Taken together these results, Immune-related genes located in the sensor and transcriptional factor layers were induced by microbial challenges, while genes comprising the intracellular-signalling parts of Toll and IMD pathways were not. These differences in induction may be due to the genes in the sensor and transcriptional factor layers only functioning when immune reactions occurred, whereas those located in intracellular-signalling parts might constantly function as non-immune signal transducers. For instance, a previous study indicates that the Toll pathway is also involved in the embryonic dorsoventral pattern signalling pathway in D. melanogaster (Morisato \& Anderson, 1995). Thus, it is likely that both Toll and IMD pathways may have roles in immune reactions as well as unknown roles, leading to results that Pelle and Tube are not only induced by microbial challenges. 

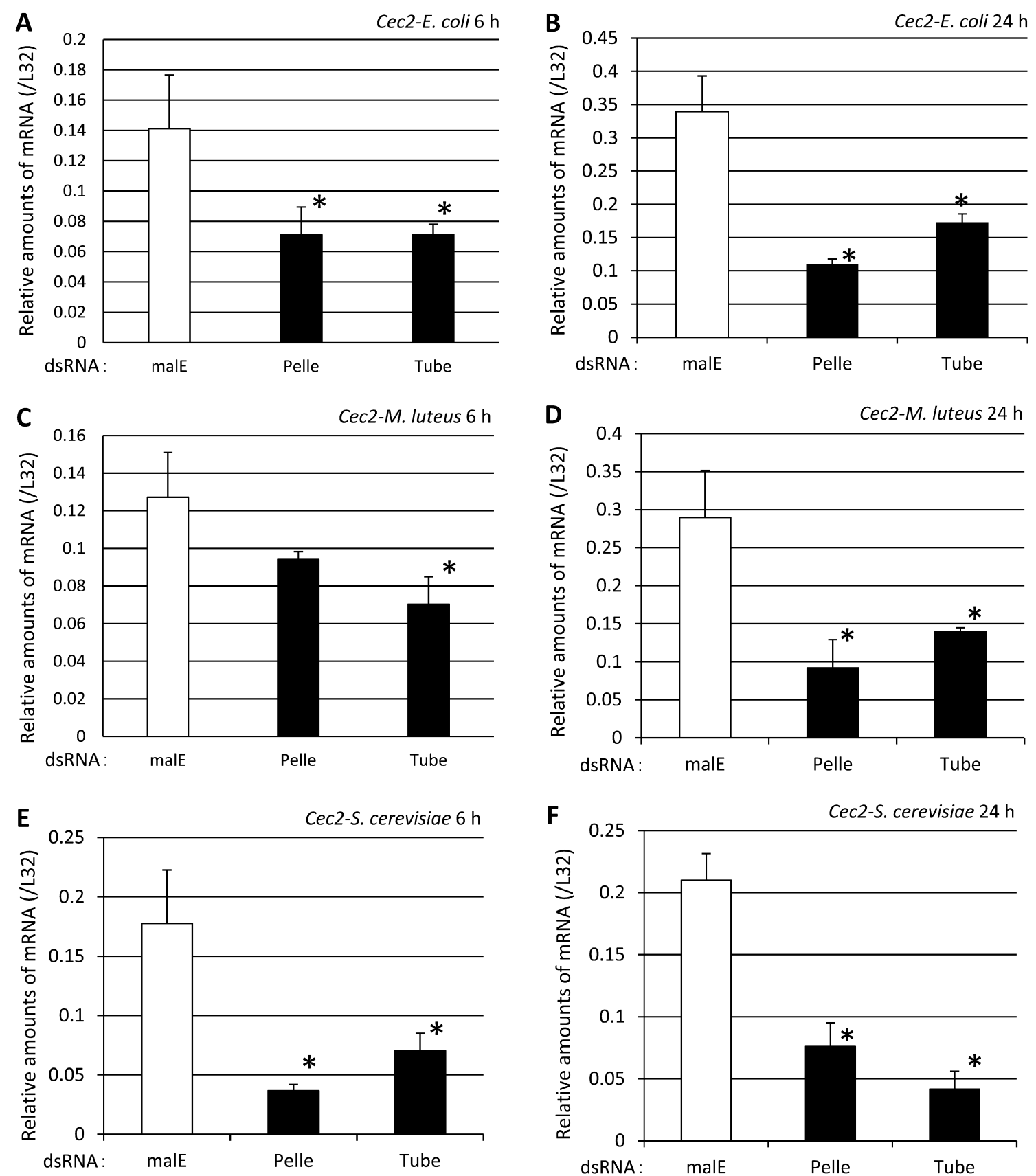

Fig. 6. Effects of the knockdown of Pelle and Tube on the microbial induction of the group III AMP gene, Cec2. 100 ng dsRNAs of Pelle, Tube and malE were injected into day 0 pupae. $72 \mathrm{~h}$ later, a heat-killed cell suspension of $E$. coli $(\mathrm{A}$ and $\mathrm{B}), M$. luteus (C and $\mathrm{D})$ or $S$. cerevisiae ( $\mathrm{E}$ and $\mathrm{F}$ ) was injected into dsRNA-treated pupae. The relative amounts of mRNA of Col1 to RpL32 were assessed $6 \mathrm{~h}$ ( $\mathrm{A}, \mathrm{C}$ and $E)$ and $24 \mathrm{~h}(\mathrm{~B}, \mathrm{D}$ and $\mathrm{F})$ after the microbial challenges. The results are the means \pm S.D. Experiments were independently repeated at least three times with three animals each. Asterisks indicate $P<0.05$ versus malE control pupae based on Student's $t$-tests.

The knockdown of Pelle or Tube altered the levels of $\mathrm{Cec} 2$ induced 6 and $24 \mathrm{~h}$ after the microbial challenges by E. coli, M. luteus and S. cerevisiae, while neither the Pelle nor Tube knockdown affected the levels of Coll, Attl or $D e f 3$ induced by the three microbial challenges, except in some combinations (Coll and Def3 in Pelle knockdown pupae by E. coli). The significantly lower level of Coll expression in Pelle knockdown pupae in response to $E$. coli challenges might be partially due to individual variants of the strength of Coll induction in pupae sampled. If Pelle mainly involved Coll induction by $E$. coli challenges, over $40 \%$ reduction in the expression levels in knockdown pupae compared with controls is expected, however, the actual reduction in the percentage of Coll is much smaller (see values in Fig. 4A and Fig. 6). Only Def3 induction by E. coli injections, both post $6 \mathrm{~h}$ and $24 \mathrm{~h}$, were altered by Pelle knockdown. One reason for this might be that Def3 inductions by $E$. coli are stronger than by $M$. luteus and $S$. cerevisiae. A previous study showed that $D e f 3$ is regulated by both IMD and Toll pathway, and Def 3 is more dependent on the IMD pathway than the Toll pathway (Yokoi et al., 2012a, b). Considering the above and the results presented in this study, the levels of induction of Def3 might be to some extent regulated mainly by the IMD pathway 

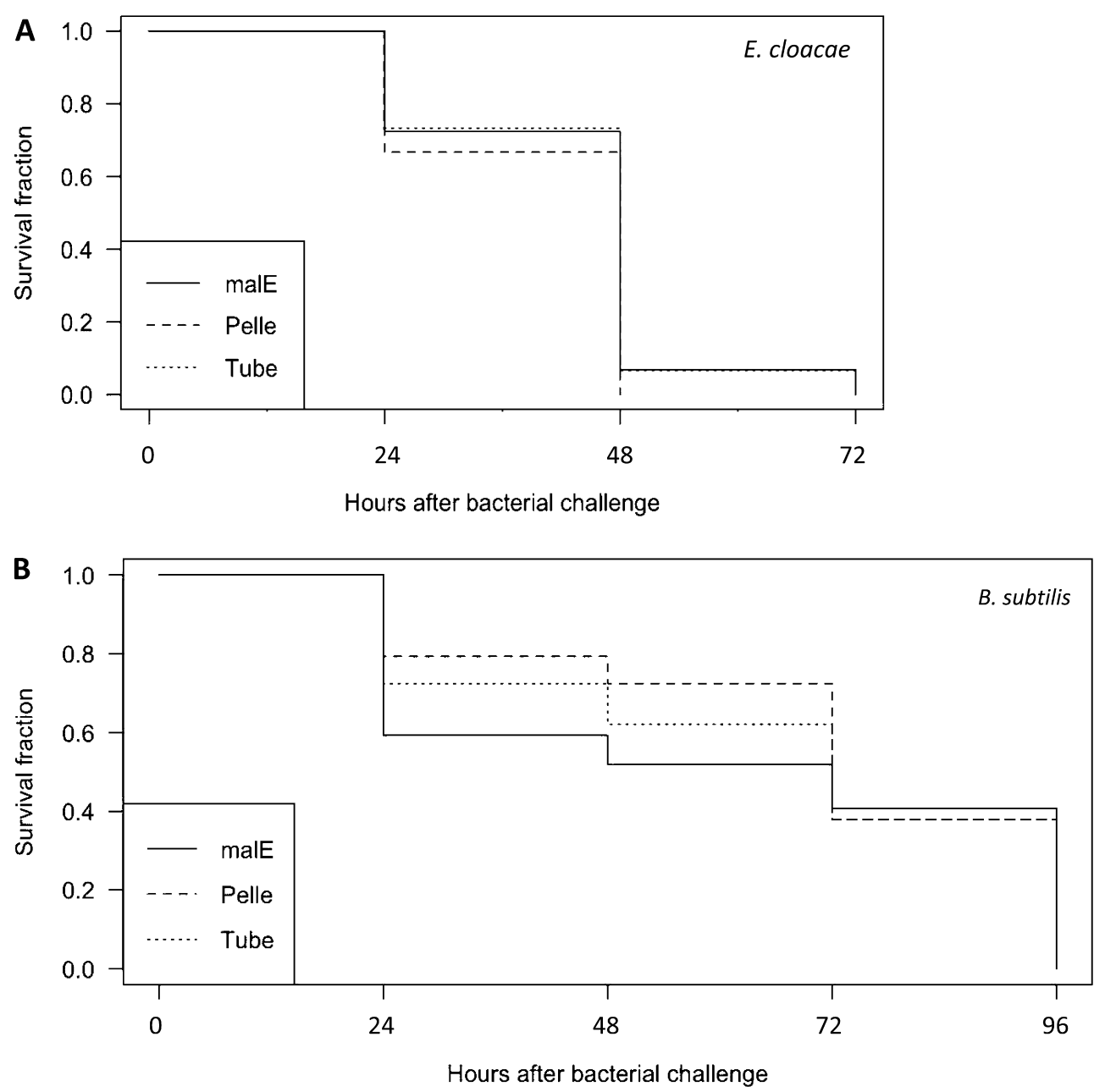

Fig. 7. Effects of the knockdown of Pelle and Tube on host defences against two model bacterial pathogens. Day 3 pupae that were preinjected with the respective dsRNAs (malE-treated pupae as controls) on day 0 were challenged by $E$. cloacae (A) or B. subtilis (B) and the survival of pupae was monitored and recorded every $24 \mathrm{~h}$. Results are shown as Kaplan-Meier plots.

and greater inductions of Def3 could be regulated by both Toll and IMD pathways. It is not clear why the variation in the induction of Def3 were recorded only for Pelle knockdown pupae. There might be a gene, which is redundant in the functioning of Tube when great levels of Def3 were induced, whereas there is no such gene with redundant functions in Pelle. The results shown in Figs 3-6 indicate that Pelle and Tube mainly contributed to the immune signal transduction via the Toll pathway as MyD88, Dif1 and Dif2 (Yokoi et al., 2012a, b). Also, these results indicate that clear immune signal crosstalk between Toll and IMD pathways via Pelle or Tube did not occur. In contrast, knockdown of the FADD and DREDD components of the IMD pathway, affected both IMD- and Toll-dependent AMP inductions, indicating that immune signal crosstalk occurred via $D R E D D$ and FADD (Yokoi et al., 2022). Thus overall, it is likely that the immune signaling via the Toll pathway reported in D. melanogaster also occurs in T. castaneum, and the gene sets involved in Toll signal transduction in $T$. castaneum do not differ significantly from those in $D$. melanogaster (Valanne et al., 2011). As described above, differences in the immune systems in terms of AMP production in $T$. castaneum and D. melanogaster involved the mechanisms underlying the activation of the Toll and IMD pathways. The Toll and IMD pathways were both activated by the three types of microbe challenges in T. castaneum, whereas the Toll pathway in D. melanogaster is mainly activated by prokaryote and Gram-positive bacterial challenges and the IMD pathway by a Gram-negative bacterial challenge. The present results support these findings, suggesting that the Toll pathway functioned as an immune signal transduction pathway induced by three types of microbial challenges transduced via the Toll pathway in $T$. castaneum.

The results of survival assays revealed that neither the Pelle nor Tube knockdown affected percentage survival following challenges with the two entomopathogenic bacteria: E. cloacae and B. subtilis. In previous reports the $M y D 88$, Dif1 or Dif2i knockdown pupae with a Toll pathway, did not succumb earlier to E. cloacae and B. subtilis infections than control pupae, whereas either IMD, FADD, DREDD or Relish knockdown pupae, with an IMD pathway, succumbed earlier to infections by E. cloacae and $B$. subtilis. Furthermore, we also showed that although Group I, II- and III-AMP genes were induced by E. cloacae and B. subtilis injections, the induction of Group III AMP 
genes were not affected by Relish knockdown (Yokoi et al., 2012a, b, 2022). Results from this study and previous reports indicate that genes related to the Toll pathway are not involved in immune reactions against these bacterial infections, whereas the genes related to the IMD pathway are involved. In D. melanogaster, adult flies with Toll pathway-related gene mutants succumbed earlier to fungi or Gram-positive bacteria (Rutschmann et al., 2002; Gottar et al., 2006), while those with IMD pathway-related gene mutants succumbed earlier to Gram-negative bacteria (Lemaitre et al., 1995; Leulier et al., 2000). These findings reflect the differences in the immune systems of $T$. castaneum and D. melanogaster and indicate that the IMD pathway plays a pivotal role in immune defences against bacterial infections in $T$. castaneum. Therefore, further studies to elucidate the immune roles of the Toll pathway in $T$. castaneum are warranted.

The present results showed that Pelle and Tube in T. castaneum were mainly involved in Toll-dependent AMP gene induction by three microbial challenges, but not in host bacterial defense reactions against two entomopathogenic bacteria. This study provides an insight into the role of the Toll pathway in this species immune system.

ACKNOWLEDGEMENTS. This work was supported by JSPS KAKENHI Grant Numbers $21 \mathrm{H} 03831$ and $21 \mathrm{~K} 19126$ to K. Yokoi, and 20K06050 to K. Miura. We also thank Y. Yagi, T. Ushimaru, A. Miyanoshita and M. Murata for providing research materials.

\section{REFERENCES}

Choe K.M., Werner T., Stöven S., Hultmark D. \& Anderson K.V. 2002: Requirement for a peptidoglycan recognition protein (PGRP) in Relish activation and antibacterial immune responses in Drosophila. - Science 296: 359-362.

Choe K.M., Lee H. \& Anderson K.V. 2005: Drosophila peptidoglycan recognition protein LC (PGRP-LC) acts as a signaltransducing innate immune receptor. - Proc. Natl. Acad. Sci. U.S.A. 102: 1122-1126.

De Gregorio E., Spellman P.T., Rubin G.M. \& Lemaitre B. 2001: Genome-wide analysis of the Drosophila immune response by using oligonucleotide microarrays. - Proc. Natl. Acad. Sci. U.S.A. 98: 12590-12595.

De Gregorio E., Spellman P.T., Tzou P., Rubin G.M. \& LemaiTRE B. 2002: The Toll and Imd pathways are the major regulators of the immune response in Drosophila. - EMBO J. 21: 2568-2579.

Ferrandon D., Imler J.L., Hetru C. \& Hoffmann J.A. 2007: The Drosophila systemic immune response: sensing and signalling during bacterial and fungal infections. - Nat. Rev. Immunol. 7: 862-874.

Gottar M., Gobert V., Matskevich A.A., Reichhart J.M., Wang C., Butt T.M., Belvin M., Hoffmann J.A. \& Ferrandon D. 2006: Dual detection of fungal infections in Drosophila via recognition of glucans and sensing of virulence factors. - Cell 127: $1425-1437$.

Guntermann S. \& Foley E. 2011: The protein Dredd is an essential component of the c-Jun N-terminal kinase pathway in the Drosophila immune response. - J. Biol. Chem. 286: 30284-30294.

HiLLYER J.F. 2016: Insect immunology and hematopoiesis. Dev. Comp. Immunol. 58: 102-118.
Horng T. \& Medzhitov R. 2001: Drosophila MyD88 is an adapter in the Toll signaling pathway. - Proc. Natl. Acad. Sci. U.S.A. 98: $12654-12658$.

Hu S. \& YANG X. 2000: dFADD, a novel death domain-containing adapter protein for the Drosophila caspase DREDD. $-J$. Biol. Chem. 275: 30761-30764.

Hultmark D. 2003: Drosophila immunity: paths and patterns. Curr. Opin. Immunol. 15: 12-19.

Ip Y.T., Reach M., Engstrom Y., Kadalayil L., Cai H., GonzÁlezCrespo S., Tatei K. \& Levine M. 1993: Dif, a dorsal-related gene that mediates an immune response in Drosophila. - Cell 75: 753-763.

Kaneko T., Yano T., Aggarwal K., Lim J.H., Ueda K., Oshima Y., Peach C., Erturk-Hasdemir D., Goldman W.E., OH B.H. et AL. 2006: PGRP-LC and PGRP-LE have essential yet distinct functions in the Drosophila immune response to monomeric DAP-type peptidoglycan. - Nat. Immunol. 7: 715-723.

Kleino A. \& Silverman N. 2014: The Drosophila IMD pathway in the activation of the humoral immune response. - Dev. Comp. Immunol. 42: 25-35.

Koyama H., Kato D., Minakuchi C., Tanaka T., Yokoi K. \& MiURA K. 2015: Peptidoglycan recognition protein genes and their roles in the innate immune pathways of the red flour beetle, Tribolium castaneum. - J. Invertebr. Pathol. 132: 86-100.

Lemaitre B. \& Hoffmann J. 2007: The host defense of Drosophila melanogaster. - Annu. Rev. Immunol. 25: 697-743.

Lemaitre B., Kromer-Metzger E., Michaut L., Nicolas E., Meister M., Georgel P., Reichhart J.M. \& Hoffmann J.A. 1995: A recessive mutation, immune deficiency (imd), defines two distinct control pathways in the Drosophila host defense. — Proc. Natl. Acad. Sci. U.S.A. 92: 9465-9469.

Lemaitre B., Nicolas E., Michaut L., Reichhart J.M. \& HoffMANN J.A. 1996: The dorsoventral regulatory gene cassette spätzle/Toll/cactus controls the potent antifungal response in Drosophila adults. - Cell 86: 973-983.

Leulier F., Rodriguez A., Khush R.S., Abrams J.M. \& Lemaitre B. 2000: The Drosophila caspase Dredd is required to resist gram-negative bacterial infection. - EMBO Rep. 1: 353-358.

Medzhitov R., Preston-Hurlburt P., Kopp E., Stadlen A., Chen C., GHosh S. \& JanewaY C.A. 1998: MyD88 is an adaptor protein in the hToll/IL-1 receptor family signaling pathways. Mol. Cell 2: 253-258.

Morisato D. \& ANDERSON K.V. 1995: Signaling pathways that establish the dorsal-ventral pattern of the Drosophila embryo. - Annu. Rev. Genet. 29: 371-399.

Rutschmann S., Kilinc A. \& Ferrandon D. 2002: Cutting edge: the toll pathway is required for resistance to gram-positive bacterial infections in Drosophila. - J. Immunol. 168: 1542-1546.

Stoven S., Silverman N., Junell A., Hedengren-Olcott M., Erturk D., Engstrom Y., Maniatis T. \& Hultmark D. 2003: Caspase-mediated processing of the Drosophila NF-kappaB factor Relish. - Proc. Natl. Acad. Sci. U.S.A. 100: 5991-5996.

Tomoyasu Y., Miller S.C., Tomita S., Schoppmeier M., GrossMANN D. \& BUCHER G. 2008: Exploring systemic RNA interference in insects: a genome-wide survey for RNAi genes in Tribolium. - Genome Biol. 9: R10, 22 pp.

Tribolium Genome Sequencing Consortium (Richards S., Gibbs R.A., Weinstock G.M., Brown S.J., Denell R., Beeman R.W., Gibbs R., Beeman R.W., Brown S.J. et AL.) 2008: The genome of the model beetle and pest Tribolium castaneum. - Nature 452: 949-955.

Valanne S., Wang J.-H. \& RÄmet M. 2011: The Drosophila Toll signaling pathway. - J. Immunol. 186: 649-656.

Wang L., Weber A.N.R., Atilano M.L., Filipe S.R., Gay N.J. \& LigoXYGakis P. 2006: Sensing of Gram-positive bacteria in 
Drosophila: GNBP1 is needed to process and present peptidoglycan to PGRP-SA. - EMBO J. 25: 5005-5014.

Weber A.N.R., Tauszig-Delamasure S., Hoffmann J.A., Lelièvre E., Gascan H., Ray K.P., Morse M.A., ImLer J.-L. \& Gay N.J. 2003: Binding of the Drosophila cytokine Spätzle to Toll is direct and establishes signaling. - Nat. Immunol. 4: 794-800.

Yoko K., Koyama H., Ito W., Minakuchi C., Tanaka T. \& Miura K. 2012a: Involvement of NF- $\mathrm{BB}$ transcription factors in antimicrobial peptide gene induction in the red flour beetle, Tribolium castaneum. - Dev. Comp. Immunol. 38: 342-351.

Yokoi K., Koyama H., Minakuchi C., Tanaka T. \& Miura K. 2012b: Antimicrobial peptide gene induction, involvement of Toll and IMD pathways and defense against bacteria in the red flour beetle, Tribolium castaneum. - Results Immunol. 2: $72-82$.

Yoко K., Ito W., Kato D. \& Miura K. 2022: RNA interferencebased characterization of Caspar, DREDD, and FADD genes in immune signaling pathways of the red flour beetle, Tribolium castaneum (Coleoptera: Tenebrionidae). - Eur. J. Entomol. 119: $23-35$
Zou Z., Evans J.D., Lu Z., Zhao P., Williams M., Sumathipala N., Hetru C., Hultmark D. \& Jiang H. 2007: Comparative genomic analysis of the Tribolium immune system. - Genome Biol. 8: R177, 16 pp.

Received December 9, 2021; revised and accepted January 11, 2022 Published online February 10, 2022

\section{Supplementary tables}

Two supplementary tables are available in figshare (DOI: 10.6084/m9.figshare.c.5529771).

\section{Supplementary Table 1}

Primer sequences for qRT-PCR (DOI: 10.6084/m9.figshare. 15059349).

\section{Supplementary Table 2}

Primer sequences for synthesizing T7-tagged cDNA (DOI: 10.6084/m9.figshare.15059367). 